\title{
EXACT DIVISORS OF POLYNOMIALS WITH PRIME VARIABLE
}

\author{
EIRA J. SCOURFIELD
}

In memory of Heini Halberstam

\begin{abstract}
In 1952 Paul Erdôs obtained upper and lower bounds of the same order of magnitude for the number $N(x)$ of divisors of an irreducible polynomial $f(n)$ with integer coefficients for $n$ up to $x$; an asymptotic formula for $N(x)$ when $f$ has degree at least 3 has not yet been established. However progress has been made in the corresponding problem when the divisors of $f(n)$ are restricted in some way and $f$ is not necessarily irreducible. In this paper we consider a polynomial $f$ with integer coefficients that may not be irreducible or squarefree. Our aim is to obtain an asymptotic formula for the number of exact divisors up to $y$ of $f(p)$ for $p$ a prime less than $x$ with $y$ as large as possible in terms of $x$. We utilize the result that Vaughan established for his elementary proof of the Bombieri-Vinogradov Theorem.
\end{abstract}

Keywords: exact divisors, polynomials with prime variable, Siegel-Walfisz theorem, BombieriVinogradov theorem.

\section{Introduction}

Let $f \in \mathbb{Z}[x]$ where $f$ is not necessarily irreducible but the degree of each irreducible factor is at least 2. Many authors have investigated problems concerning the divisors of $f(n)$ for $n \leqslant x$. A key result is due to Paul Erdôs [2] who proved using complicated elementary techniques that if $f$ is irreducible then

$$
x \log x \ll \sum_{n \leqslant x} \tau(f(n)) \ll x \log x
$$

where $\tau(k)$ is the number of positive divisors of $k$. When $f$ is an irreducible quadratic polynomial an asymptotic formula for this sum was established by Bellman and Shapiro (see [2]) and studied further in [5], [6], [7], [8], [12] but to the author's knowledge no corresponding asymptotic formula has been established for irreducible $f$ of degree at least 3 . However it is sometimes possible to derive an asymptotic formula for the number of divisors of $f(n)$ for $n \leqslant x$ satisfying an

2010 Mathematics Subject Classification: primary: 11N37; secondary: 11N64, 11N32 
additional property. When $f=\prod_{i=1}^{l} f_{i}$ where the $f_{i}$ are pairwise coprime and of degree at least 2 we proved in [13] that

$$
\sum_{n \leqslant x} \#\{m \leqslant x: m \mid f(n)\}=C x(\log x)^{l}\left(1+O\left(\frac{1}{\log x}\right)\right)
$$

where $C$ is a constant, and we obtained an asymptotic formula for the corresponding sum when $P(m):=\max _{p \mid m} p \leqslant y$, valid for $y \geqslant \exp \left((\log \log x)^{\frac{5}{3}+\epsilon}\right)$ with $\epsilon>0$, so $m$ is a smooth or friable divisor of $f(n)$. The proof of this latter result is related to and depends on ideas in [4] by Hanrot, Tenenbaum and Wu.

If $d \mid k$ and $\left(d, \frac{k}{d}\right)=1, d$ is called an exact divisor of $k$ and we write $d \| k$. In this paper we consider divisors and exact divisors up to $y=y(x)$ of $f(p)$ for a general polynomial $f$ over the integers and $p$ a prime $\leqslant x$. For the simplest case considering the divisors of $f(p)=p-a$, Linnik used his dispersion method to establish that $\sum_{a<p \leqslant x} \tau(p-a) \sim E x$ where the constant $E$ depends on $a$. Using

Bombieri's Theorem G. Rodriguez [11] and H. Halberstam [3] gave independently a much simpler proof of this result obtaining an error term $O\left(\frac{x \log \log x}{\log x}\right)$. Our results below concern polynomials

$$
f=\prod_{i=1}^{l} f_{i}^{r_{i}}
$$

where each $f_{i} \in \mathbb{Z}[x]$, is irreducible and of degree $\geqslant 2$, the $f_{i}$ are pairwise coprime, and $1 \leqslant r_{1} \leqslant \ldots \leqslant r_{l}$. Our main aim is to investigate the exact divisors $\leqslant y$ of $f(p)$ for primes $p \leqslant x$, with $y$ as large as possible in terms of $x$, but we also look at the analogous problem for the divisors of $f(p)$. As is usual in this type of problem we utilize the von Mangoldt function, defined by

$$
\Lambda(n)= \begin{cases}\log p & \text { if } n=p^{\alpha} \text { for } \alpha \geqslant 1 \\ 0 & \text { otherwise. }\end{cases}
$$

Our goal is to obtain an asymptotic formula for

$$
\sum_{M \leqslant y} \sum_{\substack{n \leqslant x \\ M \| f(n)}} \Lambda(n)
$$

for $M \in \mathbb{N}$, from which we deduce an asymptotic formula for

$$
\sum_{M \leqslant y} \sum_{\substack{p \leqslant x \\ M \| f(p)}} 1 .
$$

Our result depends on applying the Siegel-Walfisz Theorem (see Lemma 4.3) and a result of Vaughan (see Lemma 4.4 and [15]) that he used to give an essentially elementary proof of the Bombieri-Vinogradov Theorem. 
We denote a polynomial of degree $k$ by $P_{k}($.$) and assume that \varepsilon>0$ is arbitrarily small. If $r_{1} \geqslant 2, l \geqslant 1$, let

$$
\Delta=\left(1-\frac{1}{l r_{l}}+\varepsilon\right), \quad y^{\Delta} \leqslant x^{1 / 6}(\log x)^{-A-4} .
$$

If $r_{1}=1, l \geqslant 1$, let

$$
\Delta=\left(3-\frac{1}{l r_{l}}+\varepsilon\right), \quad y^{\Delta} \leqslant x^{1 / 2}(\log x)^{-A-4} .
$$

In both cases we assume that $A>0$ is the constant in Lemma 4.3 and that $y^{\frac{1}{l r_{l}}-\varepsilon} \gg(\log x)^{A+4}$.

Throughout we put

$$
E(y)=\exp \left(-(\log y)^{\frac{3}{5}-\varepsilon}\right) .
$$

Theorem 1.1. When y satisfies (1.3) or (1.4)

$$
\sum_{M \leqslant y} \sum_{\substack{p \leqslant x \\ M \| f(p)}} \log p=x\left(P_{l}(\log y)+O(E(y))+O\left(x(\log x)^{-A}\right)\right.
$$

where the leading coefficient of the polynomial $P_{l}$ is given in (3.11).

\section{Corollary 1.2.}

$$
\sum_{M \leqslant y} \sum_{\substack{p \leqslant x \\ M \| f(p)}} 1=l i(x)\left(P_{l}(\log y)+O(E(y))+O\left(x(\log x)^{-A}\right) .\right.
$$

The method used to prove Theorem 1.1 yields an analogous result when $M \|$ $f(p)$ is replaced by $M \mid f(p)$. Let $R=\sum_{i=1}^{l} r_{i}$. Suppose that for $l r_{l}>1, y$ satisfies (1.3) and for $l r_{l}=1, \Delta=2-\frac{1}{l r_{l}}+\varepsilon=1+\varepsilon$, assume that $y^{\Delta} \leqslant x^{1 / 2}(\log x)^{-A-4}$, and that in both cases $y^{\frac{1}{l r_{l}}-\varepsilon} \gg(\log x)^{A+4}$.

Theorem 1.3.

$$
\sum_{M \leqslant y} \sum_{\substack{p \leqslant x \\ M \mid f(p)}} \log p=x\left(P_{R}(\log y)+O(E(y))+O\left(x(\log x)^{-A}\right)\right.
$$

where the polynomial $P_{R}$ has degree $R$ and leading coefficient given by (6.6).

As in the Corollary 1.2, to replace the summand $\log p$ by 1 just replace $x$ by $\operatorname{li}(x)$ in the main term.

The leading coefficients of $P_{l}$ and $P_{R}$ depend on the residue of certain Dedekind zeta functions at their pole $s=1$.

We can ask the corresponding question when the prime variable $p$ is replaced by $n \in \mathbb{N}$. The error term here is much easier to obtain, and in order to derive an asymptotic formula the maximum value of $y$ can be much larger. 


\section{Theorem 1.4.}

(i) Suppose $1=r_{1}=\ldots=r_{j}<r_{j+1} \leqslant \ldots \leqslant r_{l}$ for some $j(0 \leqslant j \leqslant l)$. Then

(a)

$$
\sum_{M \leqslant y} \sum_{\substack{n \leqslant x \\ M \| f(n)}} 1=x\left(P_{l}(\log y)+O(E(y))+O\left(y(\log y)^{l+j-1}\right)\right.
$$

(b)

$$
\sum_{M \leqslant y} \sum_{\substack{n \leqslant x \\ M \| f(n)}} 1=x\left(B_{l}(\log y)^{l}+O\left((\log y)^{l-1} \log \log y\right)\right)+O\left(y(\log y)^{l-1}\right)
$$

for $j>0$ where $B_{l}$ is the leading coefficient of $P_{l}($.$) .$

(ii) With $R=\sum_{i=1}^{l} r_{i}$ as in Theorem 1.3

$$
\sum_{M \leqslant y} \sum_{\substack{n \leqslant x \\ M \mid f(n)}} 1=x\left(P_{R}(\log y)+O(E(y))+O\left(y(\log y)^{R-1}\right) .\right.
$$

We obtain an asymptotic formula in (i)(a), with main term $x P_{l}(\log y)$, when $y(\log y)^{l+j-1}=o(x)$ and in (i)(b) when $\frac{y}{\log y}=o(x)$; however in (b) we only have one term of the polynomial $P_{l}(\log y)$. When $f$ is irreducible, so $l=j=1$, and $y=x$, we obtain an asymptotic formula from (i)(b) but not from (i)(a). For an asymptotic formula with main term $x P_{R}(\log y)$ in (ii) we need $y(\log y)^{R-1}=o(x)$. For a special case of (ii) with a weaker result see (1.1) above, and for a related result with an analogous proof see Lemma 3.9 in [14].

When $f$ is an irreducible quadratic polynomial it is known that

$$
\sum_{M} \sum_{\substack{n \leqslant x \\ M \mid f(n)}} 1=C x \log x+O(x)
$$

for $C$ a constant; see [5] for a stronger result in the special case $f(n)=n^{2}+a$, and (1.1) and [13] for an arbitrary such $f$. For exact divisors of an irreducible quadratic polynomial $f$, in section 7 we deduce from Theorem 1.4(i)(b)

\section{Corollary 1.5.}

$$
\sum_{M} \sum_{\substack{n \leqslant x \\ M \| f(n)}} 1=2 B_{1} x \log x+O(x \log \log x)
$$




\section{Notation and preliminary results}

As in section 1 write $f=\prod_{i=1}^{l} f_{i}^{r_{i}} \in \mathbb{Z}[x]$ where the $f_{i}$ are irreducible, have degree $\geqslant 2$, are pairwise coprime and $1 \leqslant r_{1} \leqslant \ldots \leqslant r_{l}$. Let

$$
f_{0}=\prod_{i=1}^{l} f_{i}
$$

Throughout this paper $p, q$ denote primes, and $\varepsilon>0$ is arbitrary.

\section{Lemma 2.1.}

(i) If $q^{\alpha} \mid f_{i}^{r_{i}}(n)$ then $q^{t} \mid f_{i}(n)$ with $t=\left\lceil\frac{\alpha}{r_{i}}\right\rceil$, so $\alpha=(t-1) r_{i}+\beta_{i}$ with $t \geqslant 1$ and $1 \leqslant \beta_{i} \leqslant r_{i}$. Hence if $M_{i} \mid f_{i}^{r_{i}}(n)$ and $M_{i}=\prod_{q^{\alpha} \| M_{i}} q^{\alpha}$, then $m_{i}:=$ $\prod_{q^{\alpha} \| M_{i}} q^{t} \mid f_{i}(n)$ where $t=\left\lceil\frac{\alpha}{r_{i}}\right\rceil$.

(ii) There exists $p_{0}$ such that if $q \geqslant p_{0}$ and $q \mid f(n)$ then $q \mid f_{i}(n)$ for exactly one value of $i$. Hence if $\left(M, \prod_{q<p_{0}} q\right)=1$ and $M \mid f(n)$ then $M=\prod_{i=1}^{l} M_{i}$ where $M_{i} \mid f_{i}^{r_{i}}(n)$ for $i=1, \ldots, l$ and $\left(M_{i}, M_{j}\right)=1$ for $i \neq j$.

(iii) If $M_{i} \| f_{i}^{r_{i}}(n)$ then $m_{i}=M_{i}^{1 / r_{i}} \in \mathbb{N}$ and $m_{i} \| f_{i}(n)$. Hence in (i) each $\beta_{i}=r_{i} \quad$ and $\alpha=r_{i}$.

Proof. Part (ii) follows since if $i \neq j$ then $f_{i}$ and $f_{j}$ are coprime over $\mathbb{Q}$, and the other parts are routine.

Notation. If $\left(M, \prod_{q<p_{0}} q\right)=1$ and $M \| f(n)$ then $M=\prod_{i=1}^{l} M_{i}$ with $M_{i} \| f_{i}^{r_{i}}(n)$ and $m \| f_{0}(n)$ for $m=\prod_{i=1}^{l} m_{i}=\prod_{i=1}^{l} M_{i}^{1 / r_{i}} \in \mathbb{N}$.

Let $D_{i}$ denote the discriminant of $f_{i}$ and choose $p_{0}$ large enough for $q \nmid \prod_{i=1}^{l} D_{i}$ when $q \geqslant p_{0}$.

Define

$$
\begin{gathered}
\rho_{i}\left(m_{i}\right)=\#\left\{n\left(\bmod m_{i}\right): m_{i} \mid f_{i}(n)\right\} \\
\rho(m)=\#\left\{n(\bmod m): m \mid f_{0}(n)\right\} .
\end{gathered}
$$

\section{Lemma 2.2.}

(i) $\rho_{i}\left(m_{i}\right)$ is multiplicative. If $q \geqslant p_{0}$, then $\rho_{i}\left(q^{\alpha}\right)=\rho_{i}(q)$ for all $\alpha \geqslant 1$, and if $q<p_{0}$ then $\rho_{i}\left(q^{\alpha}\right) \ll 1$ for all $\alpha \geqslant 1$. 
(ii) If $\left(m, \prod_{q<p_{0}} q\right)=1$ then $\rho(m)=\prod_{i=1}^{l} \prod_{q \mid m_{i}} \rho_{i}(q)=\prod_{q \mid m} \rho(q)$.

Proof. See Theorems 53 and 54 in [9].

We assume throughout that $M, M_{0}$ denote positive integers satisfying

$$
\left(M, \prod_{q<p_{0}} q\right)=1, \quad\left(M_{0}, \prod_{q \geqslant p_{0}} q\right)=1,
$$

so $\left(M, M_{0}\right)=1$. Then our aim in Theorem 1.1 is to consider when $M M_{0} \| f(p)$. If $q<p_{0}$ and $l>1$, both $q \mid f_{i}(n)$ and $q \mid f_{j}(n)$ may hold for the same $n$ when $i \neq j$, so we need to treat $M_{0}$ separately from $M$.

Let

$$
\left.\begin{array}{rl}
\lambda(M) & =\#\{n(\bmod M): M \mid f(n)\} \\
\lambda\left(M_{0}\right) & =\#\left\{n\left(\bmod M_{0}\right): M_{0} \mid f(n)\right\} \\
\lambda^{*}(M) & =\#\{n(\bmod M): M \| f(n)\} .
\end{array}\right\}
$$

Lemma 2.3. If $2 \leqslant r_{1} \leqslant \ldots \leqslant r_{l}$ then

$$
\lambda^{*}(M)=\frac{M}{m} \rho(m) \prod_{q \mid m}\left(1-\frac{1}{q}\right)=\varphi(M) \frac{\rho(m)}{m} .
$$

Proof. Let $M, m, M_{i}, m_{i}$ be as in the notaion above; then if $M_{i}>1$ we see that $M_{i}>m_{i}$ since $r_{i}>1$. Let $M_{i}=\prod_{q^{\alpha} \| M_{i}} q^{\alpha}$ with $r_{i} \mid \alpha$, so $m_{i}=\prod_{q^{t} \| m_{i}} q^{t}$ with $\alpha=r_{i} t$. It follows that if $q^{\alpha} \| f_{i}^{r_{i}}(n)$ then $q^{t} \mid f_{i}(n)$ but $q^{t+1} \nmid f_{i}(n)$. Since by Lemma 2.2(i) and (2.1)

$$
\#\left\{n\left(\bmod q^{t+1}: q^{t} \| f_{i}(n)\right\}=q \rho_{i}\left(q^{t}\right)-\rho_{i}\left(q^{t+1}\right)=(q-1) \rho_{i}(q)\right.
$$

we deduce that

$$
\#\left\{n\left(\bmod q^{\alpha}: q^{\alpha} \| f_{i}^{r_{i}}(n)\right\}=q^{\alpha-(t+1)}(q-1) \rho_{i}(q)=q^{\alpha-t}\left(1-\frac{1}{q}\right) \rho_{i}(q) .\right.
$$

Hence by multiplicity

$$
\lambda^{*}(M)=\prod_{i=1}^{l} \frac{M_{i}}{m_{i}} \prod_{q \mid M_{i}}\left(1-\frac{1}{q}\right) \rho_{i}(q)=\varphi(M) \frac{\rho(m)}{m} .
$$

We treat the case $r_{1}=1$ when $M_{1}=m_{1}$ differently from that of $r_{1}>1$. We observe that if $M \| f(n)$ then $M \mid f(n)$ but for all squarefree $k>1$ with $k \mid M$ we have $M k \nmid f(n)$.

Lemma 2.4. Let $r_{1}=1$ and $k$ be as above. Then

$$
\lambda(M k)=\lambda(M)=\frac{M}{m} \rho(m) .
$$


Proof. If $r_{i} \mid \alpha$ and $q^{\alpha+1} \mid f_{i}^{r_{i}}(n)$ then $q^{\frac{\alpha}{r_{i}}+1} \mid f_{i}(n)$. With the above notation, it follows that $M k|f(n) \Leftrightarrow m k| f_{0}(n)$, and hence

$$
\begin{aligned}
\lambda(M k) & =\#\left\{n(\bmod M k): m k \mid f_{0}(n)\right\} \\
& =\frac{M k}{m k} \#\left\{n(\bmod m k): m k \mid f_{0}(n)\right\}=\frac{M}{m} \rho(m)
\end{aligned}
$$

since $\rho(m k)=\prod_{q \mid m k} \rho(q)=\rho(m)$ as $k \mid m$.

Note: If $r_{i} \nmid \alpha$ and $q^{\alpha+1} \mid f_{i}^{r_{i}}(n)$ then $q^{\left\lceil\alpha / r_{i}\right\rceil} \mid f_{i}(n)$ so the above argument fails.

\section{Lemma 2.5.}

$$
\lambda\left(M_{0}\right) \ll M_{0}^{1-\frac{1}{l r_{l}}} \prod_{q^{\alpha} \| M_{0}} e^{c \sqrt{\alpha}}
$$

where $c=\pi \sqrt{\frac{2}{3}}$.

Proof. If $q \mid M_{0}$ and $l>1$, we observed that both $q \mid f_{i}(n)$ and $q \mid f_{j}(n)$ may hold when $i \neq j$. However if $q^{\alpha} \mid f(n)$ then $q^{\alpha_{i}} \mid f_{i}^{r_{i}}(n)$ for $i=1, \ldots, l$ with $\sum_{i=1}^{l} \alpha_{i}=\alpha$. Given $\alpha \geqslant 1$ the number of such sets $\left\{\alpha_{1}, \ldots, \alpha_{l}\right\}$ is at most $l ! p(\alpha) \ll e^{c \sqrt{\alpha}}, c=$ $\pi \sqrt{\frac{2}{3}}$, where $p(\alpha)$ denotes the number of partitions of $\alpha$ into positive integers; see Theorem 10.12 in [10]. If $q<p_{0}$ and $q^{\alpha} \mid f(n)$ with $\alpha \geqslant 1$ then for at least one partition of $\alpha$ of the above type

$$
\begin{aligned}
0 & <\#\left\{n\left(\bmod q^{\alpha}\right): q^{\alpha_{i}} \mid f_{i}^{r_{i}}(n), i=1, \ldots, l .\right\} \\
& \leqslant \min _{\alpha_{i} \geqslant 1,1 \leqslant i \leqslant l} \#\left\{n\left(\bmod q^{\alpha}\right): q^{\alpha_{i}} \mid f_{i}^{r_{i}}(n)\right\} \\
& \ll \min _{\alpha_{i} \geqslant 1,1 \leqslant i \leqslant l} q^{\alpha-\left\lceil\alpha_{i} / r_{i}\right\rceil} \rho_{i}\left(q^{\left\lceil\alpha_{i} / r_{i}\right\rceil}\right) \ll q^{\alpha\left(1-\frac{1}{l r_{l}}\right)}
\end{aligned}
$$

since $\left\lceil\alpha_{i} / r_{i}\right\rceil \geqslant \alpha_{i} / r_{i}, \quad \max _{1 \leqslant i \leqslant l} \alpha_{i} \geqslant \alpha / l$ and $r_{i} \leqslant r_{l}$. Hence $\lambda\left(q^{\alpha}\right) \ll q^{\alpha\left(1-\frac{1}{l r_{l}}\right)} e^{c \sqrt{\alpha}}$, and the result of Lemma 2.5 follows for $l>1$. When $l=1$, (2.7) holds since $\lambda\left(M_{0}\right) \ll M_{0}^{1-\frac{1}{r_{1}}}$.

Corollary 2.6. Let $M, k, M_{0}$ be as above and $k_{0}$ be a squarefree divisor of $M_{0}$. Then

$$
\lambda\left(M k M_{0} k_{0}\right)=\lambda(M) \lambda\left(M_{0} k_{0}\right)
$$

where $\lambda\left(M_{0} k_{0}\right) \ll M_{0}^{1-\frac{1}{l r_{l}}+\varepsilon}$. 


\section{Generating functions}

Let $K_{i}=\mathbb{Q}\left(\theta_{i}\right)$ where $f_{i}\left(\theta_{i}\right)=0$, so $\theta_{i} \notin \mathbb{Q}$. For $\sigma=\operatorname{Re} s>1$ the Dedekind zeta function $\zeta_{i}(s)$ associated with $K_{i}$ is defined by

$$
\zeta_{i}(s)=\sum_{\mathfrak{a}}(N(\mathfrak{a}))^{-s}=\prod_{\mathfrak{p}}\left(1-(N(\mathfrak{p}))^{-s}\right)^{-1}
$$

where $\mathfrak{a}$ denotes an ideal and $\mathfrak{p}$ a prime ideal of $K_{i}$ and $N($.$) denotes the norm.$ $\zeta_{i}(s)$ has a simple pole at $s=1$ with residue that we denote by $\lambda_{i}$.

Lemma 3.1. For a suitable choice of $p_{0}$ and for $\sigma>1$

$$
\zeta_{i}(s)=\prod_{q \geqslant p_{0}}\left(1-q^{-s}\right)^{-\rho_{i}(q)} h_{i}(s)
$$

where $h_{i}(s)$ is analytic in $\sigma>\frac{1}{2}$ and $h_{i}(1)>0$.

This is well known; for example, see Lemma 2 and equation (2.19) of [13].

Suppose that $F(s)$ is a product of $j$ Dedekind zeta functions (not necessarily distinct), each having a simple pole at $s=1$, together with a function $H(s)$ analytic in $\sigma>1-\delta$ for some $\delta \quad(0<\delta<1)$. We assume that the Dirichlet series for $F(s)$ in $\sigma>1$ is of the form

$$
F(s)=\sum_{n=1}^{\infty} n a(n) n^{-s}
$$

where $a(n)$ is multiplicative and non-negative. We consider several such functions below and we require estimates for

$$
S(y):=\sum_{n \leqslant y} n a(n), \quad T(y):=\sum_{n \leqslant y} a(n) .
$$

Lemma 3.2.

$$
S(y)=y P_{j-1}(\log y)+O(y E(y))
$$

where $P_{j-1}$ is a polynomial of degree $j-1$ and $E(y)$ is given by (1.5).

$$
T(y)=P_{j}(\log y)+O(E(y))
$$

where $P_{j}$ has degree $j$ and leading coefficient

$$
\frac{1}{j !} \lim _{s \rightarrow 1}\left((s-1)^{j} F(s)\right) \text {. }
$$

Proof. This follows by standard analytical methods, starting from

$$
\frac{1}{y} \int_{1-}^{y} S(u) d u=\frac{1}{2 \pi i} \int_{\kappa-i \infty}^{\kappa+i \infty} F(s) \frac{y^{s}}{s(s+1)} d s=y P_{j-1}(\log y)+O(y E(y))
$$

with $\kappa=1+\frac{1}{\log y}$, and then using that $S(u)$ in (3.4) is non-decreasing to obtain (3.5) and partial summation to deduce (3.6). 
Let $M, M_{0}$ be as in (2.3), $m$ be as in the notation and $k_{0} \mid M_{0}$ be squarefree.

\section{Lemma 3.3.}

$$
\sum_{M M_{0} \leqslant y} \frac{\rho(m)}{m} \frac{1}{\varphi\left(M_{0}\right)} \sum_{k_{0} \mid M_{0}} \frac{\mu\left(k_{0}\right)}{k_{0}} \lambda\left(M_{0} k_{0}\right)=P_{l}(\log y)+O(E(y))
$$

where the polynomial $P_{l}$ of degree $l$ has leading coefficient given in (3.11).

Proof. Let

$$
\begin{aligned}
F_{1}(s) & :=\sum_{M} \frac{M}{m} \rho(m) M^{-s}=\prod_{i=1}^{l} \prod_{q \geqslant p_{0}}\left(1+\rho_{i}(q) \sum_{t=1}^{\infty} q^{t\left(r_{i}-1\right)-t r_{i} s}\right) \\
& =\prod_{i=1}^{l} \zeta_{i}\left(r_{i}(s-1)+1\right) H_{1}(s)
\end{aligned}
$$

on using (3.2), where $H_{1}(s)$ is analytic in $\sigma>1-\frac{1}{2 r_{l}}$. Let

$$
G_{1}(s)=\sum_{M_{0}} \frac{M_{0}}{\varphi\left(M_{0}\right)} \sum_{k_{0} \mid M_{0}} \frac{\mu\left(k_{0}\right)}{k_{0}} \lambda\left(M_{0} k_{0}\right) M_{0}^{-s} .
$$

The coefficient of $M_{0}^{-s}$ is $\ll M_{0}^{1-\frac{1}{l r_{l}}+\varepsilon}$ by Lemma 2.5 and since the sum over $k_{0}$ has a bounded number of terms so $M_{0} / \varphi\left(M_{0}\right) \ll 1$. Hence $G_{1}(s)$ is analytic in $\sigma>1-\frac{1}{l r_{l}}+\varepsilon$.

We now apply Lemma 3.2(ii) to obtain (3.8) with the function in (3.3) given by $F(s)=F_{1}(s) G_{1}(s)$; by (3.7) the leading coefficient of $P_{l}$ is

$$
\begin{aligned}
\frac{1}{l !} \lim _{s \rightarrow 1} \prod_{i=1}^{l}\left((s-1) \zeta_{i}\left(r_{i}(s-1)+1\right)\right) H_{1}(s) G_{1}(s) & \\
& =\frac{1}{l !} \prod_{i=1}^{l} \frac{\lambda_{i}}{r_{i}} H_{1}(1) G_{1}(1) .
\end{aligned}
$$

By a similar argument we have:

\section{Lemma 3.4.}

$$
\sum_{M M_{0} \leqslant y} \frac{\rho(m)}{m} \prod_{q \mid m} \frac{1+\frac{1}{q}}{1-\frac{1}{q}} \frac{1}{\varphi\left(M_{0}\right)} M_{0}^{1-\frac{1}{l r_{l}}+\varepsilon} \ll(\log y)^{l} .
$$

Lemma 3.5. Let $\omega(m)$ denote the number of distinct primes dividing $m$.

$$
\sum_{M M_{0} \leqslant y} \frac{M}{m} \rho(m) 2^{\omega(m)} \lambda\left(M_{0}\right) \ll y(\log y)^{2 l-1} .
$$


Proof. In this case the generating function $F_{1}(s)$ in (3.9) is replaced by

$$
F_{2}(s)=\prod_{i=1}^{l} \prod_{q \geqslant p_{0}}\left(1+2 \rho_{i}(q) \sum_{t=1}^{\infty} q^{t\left(r_{i}-1\right)-t r_{i} s}\right)=\prod_{i=1}^{l} \zeta_{i}^{2}\left(r_{i}(s-1)+1\right) H_{2}(s)
$$

with $H_{2}(s)$ analytic in $\sigma>1-\frac{1}{2 r_{l}}$, and instead of $G_{1}(s)$ in (3.10) we use

$$
G_{2}(s)=\sum_{M_{0}} \lambda\left(M_{0}\right) M_{0}^{-s}
$$

Now apply Lemma 3.2(ii) again to obtain (3.13).

\section{Lemma 3.6.}

$$
M \frac{\rho(m)}{m} 2^{\omega(m)} \ll M^{1-\frac{1}{r_{l}}+\varepsilon} .
$$

Proof. Let $d=\operatorname{deg} f_{0}$. Then since $\rho(q) \leqslant d$ for each prime $q \geqslant p_{0}, \rho(m) \leqslant d^{\omega(m)}$. We now use that

$$
\omega(m) \leqslant \frac{\log m}{\log _{2} m}\left(1+O\left(\frac{1}{\log _{2} m}\right)\right), \quad M^{1 / r_{l}} \leqslant m=\prod_{i=1}^{l} M_{i}^{1 / r_{i}} \leqslant M^{1 / r_{1}}
$$

to deduce that

$$
\begin{aligned}
\frac{M}{m} \rho(m) 2^{\omega(m)} & \leqslant M^{1-\frac{1}{r_{l}}} \exp \left(\log (2 d) \frac{\log m}{\log _{2} m}\left(1+O\left(\frac{1}{\log _{2} m}\right)\right)\right) \\
& \leqslant M^{1-\frac{1}{r_{l}}} \exp \left(\frac{\log (2 d)}{r_{1}} \frac{\log M}{\log _{2} M}\left(1+O\left(\frac{1}{\log _{2} M}\right)\right)\right)
\end{aligned}
$$

and now (3.14) follows.

\section{Properties of the $\Psi$ function}

The von Mangoldt function $\Lambda(n)$ is defined in (1.2). Let $\chi$ denote a character and $\chi_{0}$ the principal character $(\bmod K)$ and suppose $(b, K)=1$. Define

$$
\begin{gathered}
\Psi(x ; b, K)=\sum_{\substack{n \leqslant x \\
n \equiv b(\bmod K)}} \Lambda(n), \\
\Psi(x ; \chi)=\sum_{n \leqslant x} \Lambda(n) \chi(n) .
\end{gathered}
$$

Lemma 4.1. Let $\chi(\bmod K)$ be induced by the primitive character $\chi^{\prime}$. Then

$$
\Psi(x ; \chi)=\Psi\left(x ; \chi^{\prime}\right)+O\left((\log (x K))^{2}\right) .
$$




\section{Lemma 4.2.}

$$
\Psi(x ; b, K)=\frac{x}{\varphi(K)}+O\left(\frac{1}{\varphi(K)}\left(\sum_{\chi \neq \chi_{0}}|\Psi(x ; \chi)|+x E(x)\right)\right)
$$

where $E(x)$ is given by (1.5).

For these two lemmas see, for example, section 28 of [1] where the argument after equation (2) includes an extra term $\Psi\left(x ; \chi_{0}\right)-x$ in the sum on the right of (4.4) above and this is $\ll x E(x)$ by the prime number theorem.

Lemma 4.3 (Siegel-Walfisz Theorem). Let $\chi$ be a non-principal character $(\bmod K)$ and suppose $K \leqslant(\log x)^{A}$ for some $A>0$. Then there exists an ineffective constant $C(A)$ such that

$$
\Psi(x ; \chi) \ll_{A} x \exp \left(-C(A)(\log x)^{1 / 2}\right) .
$$

See, for example, equation (3) of section 22 in [1].

Lemma 4.4. Let $\sum_{\chi}^{*}$ denote the sum over all non-principal primitive characters $\chi(\bmod K)$. For $Y \geqslant 1, \quad x \geqslant 2$

$$
\sum_{K \leqslant Y} \frac{K}{\varphi(K)} \sum_{\chi X \leqslant x}^{*} \max _{\chi}|\Psi(X ; \chi)| \ll\left(x+x^{5 / 6} Y+x^{1 / 2} Y^{2}\right)\left(\log (x Y)^{4} .\right.
$$

See, for example, the main Theorem in [15], or equation (2) in section 28 of [1]. Our aim is to investigate the sum

$$
\sum_{M M_{0} \leqslant y} \sum_{\substack{p^{\alpha} \leqslant x \\ M M_{0} \| f(p)}} \Lambda\left(p^{\alpha}\right) .
$$

We assume $M, M_{0}$ satisfy (2.3) and consider separately the cases $r_{1}>1, r_{1}=1$.

Let $\delta=\frac{1}{l r_{l}}-\varepsilon>0$, so $0<\delta<\frac{1}{2}$ if $l r_{l} \geqslant 2$ and $\delta=1-\varepsilon$ when $r_{l}=l=1$. Assume in the next two lemmas that $Y^{\delta}>(\log x)^{A+4}$ with $A$ as in Lemma 4.3.

Lemma 4.5. For $0<\delta<\frac{1}{2}$ suppose $Y^{1-\delta} \leqslant x^{1 / 6}(\log x)^{-A-4}$ with $A>0$. Then

$$
\sum_{K \leqslant Y} \frac{K^{1-\delta}}{\varphi(K)} \sum_{\chi}^{*}|\Psi(x, \chi)| \ll x(\log x)^{-A} .
$$

When $\delta=1-\varepsilon$ with $\varepsilon$ positive and small, then (4.7) holds for $Y^{1+\varepsilon} \leqslant$ $x^{1 / 2}(\log x)^{-A-4}$.

Proof. Let $W=2^{k}$ for $k$ in the range $(\log x)^{\frac{A+4}{\delta}}<2^{k} \leqslant Y$. Then by (4.6)

$$
\begin{aligned}
\sum_{W<K \leqslant 2 W} \frac{K^{1-\delta}}{\varphi(K)} \sum_{\chi}^{*}|\Psi(x, \chi)| & \ll W^{-\delta} \sum_{W<K \leqslant 2 W} \frac{K}{\varphi(K)} \sum_{\chi}^{*}|\Psi(x, \chi)| \\
& \ll W^{-\delta}\left(x+x^{5 / 6} W+x^{1 / 2} W^{2}\right)(\log (x W))^{4} .
\end{aligned}
$$


Summing (4.8) over $k$ in the range given above we find that

$$
\begin{aligned}
\sum_{(\log x)^{(A+4) / \delta}<K \leqslant Y} & \frac{K^{1-\delta}}{\varphi(K)} \sum_{\chi}^{*}|\Psi(x, \chi)| \\
& \ll\left(\frac{x}{(\log x)^{A+4}}+x^{5 / 6} Y^{1-\delta}+x^{1 / 2} Y^{2-\delta}\right)(\log (x Y))^{4} .
\end{aligned}
$$

For $0<\delta<\frac{1}{2}, \quad \min \left(x^{1 / 6(1-\delta)}, x^{1 / 2(2-\delta)}\right)=x^{1 / 6(1-\delta)}$ and for $\delta=1-\varepsilon$, $\min \left(x^{1 / 6(1-\delta)}, x^{1 / 2(2-\delta)}\right)=x^{1 / 2(1+\varepsilon)}$, so the right side of $(4.9)$ is $\ll x(\log x)^{-A}$ when $Y$ satisfies the conditions in the lemma.

By (4.5) with $A$ replaced by $(A+4) / \delta$, we deduce, since $\sum_{\chi}^{*}$ has at most $\varphi(K)$ terms, that

$$
\begin{aligned}
\sum_{K \leqslant(\log x)^{(A+4) / \delta}} \frac{K^{1-\delta}}{\varphi(K)} & \sum_{\chi}^{*}|\Psi(x, \chi)| \\
& \ll(\log x)^{(A+4)(2-\delta) / \delta} x \exp \left(-C((A+4) / \delta)(\log x)^{1 / 2}\right) \\
& \ll x(\log x)^{-A}
\end{aligned}
$$

for $x$ sufficiently large. The result of the Lemma 4.5 now follows from (4.9) and (4.10).

Lemma 4.6. For $0<\delta<1$ and $Y^{3-\delta} \leqslant x^{1 / 2}(\log x)^{-A-4}$

$$
Y^{1-\delta} \sum_{Y<K \leqslant Y^{2}} \frac{1}{\varphi(K)} \sum_{\chi}^{*}|\Psi(x, \chi)| \ll x(\log x)^{-A} .
$$

Proof. Let $W=2^{k}$ for $k$ in the range $Y<2^{k} \leqslant Y^{2}$. Then by (4.6)

$$
\sum_{W<K \leqslant 2 W} \frac{1}{\varphi(K)} \sum_{\chi}^{*}|\Psi(x, \chi)| \ll\left(\frac{x}{W}+x^{5 / 6}+x^{1 / 2} W\right)(\log (x W))^{4} .
$$

Summing (4.12) over $k$ in the range given above we have

$$
Y^{1-\delta} \sum_{Y<K \leqslant Y^{2}} \frac{1}{\varphi(K)} \sum_{\chi}^{*}|\Psi(x, \chi)| \ll Y^{1-\delta}\left(\frac{x}{Y}+x^{5 / 6} \log Y+x^{1 / 2} Y^{2}\right)(\log (x Y))^{4} .
$$

Since $\min \left(x^{1 / 6(1-\delta)}, x^{1 / 2(3-\delta)}\right)=x^{1 / 2(3-\delta)}$ for all $\delta$ with $0<\delta<1$, (4.11) follows from (4.13).

Corollary 4.7. For $0<\delta<1$ and $Y^{3-\delta} \leqslant x^{1 / 2}(\log x)^{-A-4}$

$$
\sum_{K \leqslant Y^{2}} \frac{\min \left(K^{1-\delta}, Y^{1-\delta}\right)}{\varphi(K)} \sum_{\chi}^{*}|\Psi(x, \chi)| \ll x(\log x)^{-A}
$$


This follows from Lemmas 4.5 and 4.6 , and is required when $r_{1}=1$. When $r_{1}>1$, Lemma 4.5 suffices.

When $2 \leqslant r_{1} \leqslant \ldots \leqslant r_{l}$ we see by Lemma 2.3 that $M \| f(n)$ for $n$ lying in $\lambda^{*}(M)$ residue classes $(\bmod M)$. Let $k_{0} \mid M_{0}$ be squarefree, and let $b_{j}, \quad 1 \leqslant j \leqslant$ $\lambda^{*}(M) \lambda\left(M_{0} k_{0}\right)$, denote the residue classes $\left(\bmod M M_{0} k_{0}\right)$ for which

$$
M \| f(n), \quad M_{0} k_{0} \mid f(n) .
$$

Lemma 4.8. When $2 \leqslant r_{1} \leqslant \ldots \leqslant r_{l}$

$$
\sum_{\substack{n \leqslant x \\ M M_{0} \| f(n)}} \Lambda(n)=\sum_{k_{0} \mid M_{0}} \mu\left(k_{0}\right) \sum_{j=1}^{\lambda^{*}(M) \lambda\left(M_{0} k_{0}\right)} \sum_{\substack{n \leqslant x \\ n \equiv b_{j}\left(\bmod M M_{0} k_{0}\right)}} \Lambda(n) .
$$

Now suppose $r_{1}=1$ and $k\left|M, \quad k_{0}\right| M_{0}$ are both squarefree. As in the remark before Lemma 2.4 $M M_{0} \| f(p) \Longleftrightarrow M M_{0} \mid f(p)$ but $M k M_{0} k_{0} \nmid f(p)$ for all squarefree $k k_{0}>1$. By $(2.8),(2.6)$ and $(2.7)$

$$
\lambda\left(M k M_{0} k_{0}\right)=\lambda(M) \lambda\left(M_{0} k_{0}\right)=\frac{M}{m} \rho(m) \lambda\left(M_{0} k_{0}\right)
$$

where $\lambda\left(M_{0} k_{0}\right) \ll M_{0}^{1-\frac{1}{l r_{l}}+\varepsilon}$ since $k_{0} \leqslant \prod_{q<p_{0}} q \ll 1$. Let $b_{j}, \quad 1 \leqslant j \leqslant \lambda\left(M k M_{0} k_{0}\right)$, denote the residue classes $\left(\bmod M k M_{0} k_{0}\right)$ for which $M k M_{0} k_{0} \mid f(n)$, so $b_{j}$ depends on $k, k_{0}$ as well as $M, M_{0}$.

Lemma 4.9. When $r_{1}=1$

$$
\sum_{\substack{n \leqslant x \\ M M_{0} \| f(n)}} \Lambda(n)=\sum_{\substack{k\left|M \\ k_{0}\right| M_{0}}} \mu(k) \mu\left(k_{0}\right) \sum_{j=1}^{\lambda(M k) \lambda\left(M_{0} k_{0}\right)} \sum_{\substack{n \leqslant x \\ n \equiv b_{j}\left(\bmod M k M_{0} k_{0}\right)}} \Lambda(n)
$$

Lemmas 4.8 and 4.9 follow from above and the inclusion-exclusion principle.

We note that

$$
\sum_{\substack{n \leqslant x \\ M M_{0} \| f(n)}} \Lambda(n)-\sum_{\substack{p^{\alpha} \leqslant x \\ M M_{0} \| f(p)}} \Lambda\left(p^{\alpha}\right) \ll \sum_{\substack{\alpha \leqslant x \\ p^{\alpha} \leqslant 2 \\ \alpha \geqslant 2}} \Lambda\left(p^{\alpha}\right) \ll \sqrt{x}
$$




\section{Proof of Theorem 1.1}

Case 1: $2 \leqslant r_{1} \leqslant \ldots \leqslant r_{l}$

By (4.4), (4.17) and Lemma 4.8

$$
\begin{aligned}
\sum_{\substack{p \leqslant x \\
M M_{0} \| f(p)}} \log p & =\sum_{\substack{n \leqslant x \\
M M_{0} \| f(n)}} \Lambda(n)+O(\sqrt{x}) \\
& =\sum_{k_{0} \mid M_{0}} \mu\left(k_{0}\right) \sum_{j=1}^{\lambda^{*}(M) \lambda\left(M_{0} k_{0}\right)} \Psi\left(x ; b_{j}, M M_{0} k_{0}\right)+O(\sqrt{x}) \\
& =x \frac{\lambda^{*}(M)}{\varphi(M)} \sum_{k_{0} \mid M_{0}} \mu\left(k_{0}\right) \frac{\lambda\left(M_{0} k_{0}\right)}{\varphi\left(M_{0} k_{0}\right)}+\mathcal{E}\left(x ; M, M_{0}\right)
\end{aligned}
$$

where by Lemmas 4.1 and 4.2

$$
\begin{aligned}
\mathcal{E}\left(x ; M, M_{0}\right) & \\
\ll & \lambda^{*}(M) \sum_{k_{0} \mid M_{0}}\left|\mu\left(k_{0}\right)\right| \lambda\left(M_{0} k_{0}\right) \\
& \times\left\{\frac{1}{\varphi\left(M M_{0} k_{0}\right)}\left(\sum_{\chi}^{*}|\Psi(x ; \chi)|+x E(x)\right)+\left(\log \left(x M M_{0} k_{0}\right)\right)^{2}\right\} \\
& +\sqrt{x}
\end{aligned}
$$

with $\sum_{\chi}^{*}$ defined in Lemma 4.4 and $\chi$ denoting a character $\left(\bmod M M_{0} k_{0}\right)$. The sum over $k_{0}$ in (5.1) and (5.2) has a bounded number of terms since $q \mid k_{0} \Longrightarrow q<p_{0}$, and by $(2.7) \lambda\left(M_{0} k_{0}\right) \ll M_{0}^{1-\frac{1}{l r_{l}}+\varepsilon}$ on using that $k_{0} \ll 1$ for squarefree $k_{0} \mid M_{0}$. Hence

$$
C\left(M_{0}\right):=\frac{1}{\varphi\left(M_{0}\right)} \sum_{k_{0} \mid M_{0}} \frac{\mu\left(k_{0}\right)}{k_{0}} \lambda\left(M_{0} k_{0}\right) \ll M_{0}^{-\frac{1}{l r_{l}}+\varepsilon} .
$$

By (2.5) the coefficient of $x$ in the main term of (5.1) is $\frac{\rho(m)}{m} C\left(M_{0}\right)$. By (3.8)

$$
\sum_{M M_{0} \leqslant y} \frac{\rho(m)}{m} C\left(M_{0}\right)=P_{l}(\log y)+O(E(y))
$$

where the leading coefficient of the polynomial $P_{l}$ is given in (3.11).

It remains to estimate $\sum_{M M_{0} \leqslant y}\left|\mathcal{E}\left(x ; M, M_{0}\right)\right|$ which we split into several parts.

(i) For squarefree $k_{0} \mid M_{0}, k_{0} \leqslant \prod_{q \mid M_{0}} q=c_{0} \ll 1$, so $M M_{0} \leqslant y \Rightarrow M M_{0} k_{0} \leqslant$ $c_{0} y$. By $(2.5),(2.7)$ and $(3.14) \lambda^{*}(M) \lambda\left(M_{0} k_{0}\right) \ll\left(M M_{0}\right)^{1-\delta}$ where $\delta=$ 
$\frac{1}{l r_{l}}-\varepsilon$. Hence with $K=M M_{0} k_{0}$ and $Y=c_{0} y$, we have by (4.7) provided $Y^{1-\delta} \leqslant x^{1 / 6}(\log x)^{-A-4}$ that

$$
\begin{aligned}
\sum_{M M_{0} \leqslant y} \lambda^{*}(M) \sum_{k_{0} \mid M_{0}}\left|\mu\left(k_{0}\right)\right| \lambda\left(M_{0} k_{0}\right) \frac{1}{\varphi\left(M M_{0} k_{0}\right)} \sum_{\chi}^{*}|\Psi(x ; \chi)| \\
\ll \sum_{K \leqslant Y} \frac{K^{1-\delta}}{\varphi(K)} \sum_{\chi}^{*}|\Psi(x ; \chi)| \ll x(\log x)^{-A}
\end{aligned}
$$

where $\chi$ denotes a primitive character $(\bmod K)$.

(ii) By (2.5) and (3.12)

$$
\begin{aligned}
x E(x) \sum_{M M_{0} \leqslant y} \frac{\lambda^{*}(M)}{\varphi(M)} \sum_{k_{0} \mid M_{0}}\left|\mu\left(k_{0}\right)\right| & \lambda\left(M_{0} k_{0}\right) \frac{1}{\varphi\left(M_{0} k_{0}\right)} \\
& \ll x E(x) \sum_{M M_{0} \leqslant y} \frac{\rho(m)}{m} \frac{\lambda\left(M_{0}\right)}{\varphi\left(M_{0}\right)} \\
& \ll x E(x)(\log y)^{l} .
\end{aligned}
$$

(iii)

$$
\begin{aligned}
\sum_{M M_{0} \leqslant y} \lambda^{*}(M) \sum_{k_{0} \mid M_{0}}\left|\mu\left(k_{0}\right)\right| \lambda\left(M_{0} k_{0}\right)\left(\log \left(x M M_{0} k_{0}\right)\right)^{2} \\
\ll(\log (x y))^{2} \sum_{M M_{0} \leqslant y} \varphi(M) \frac{\rho(m)}{m} \lambda\left(M_{0}\right) \\
\ll(\log (x y))^{2} y(\log y)^{l-1}
\end{aligned}
$$

on adapting the proof of (3.13) by removing the factor $2^{\omega(m)}$.

On combining (5.2), (5.5), (5.6) and (5.7) and assuming (1.3) we find that

$$
\begin{aligned}
\sum_{M M_{0} \leqslant y} \mid \mathcal{E} & \left(x ; M, M_{0}\right) \mid \\
& \ll x(\log x)^{-A}+x E(x)(\log y)^{l}+(\log (x y))^{2} y(\log y)^{l-1}+\sqrt{x} y \\
& \ll x(\log x)^{-A} .
\end{aligned}
$$

From (5.1), (5.4) and (5.8) we obtain under the assumption (1.3) that when $r_{1}>1$

$$
\sum_{M M_{0} \leqslant y} \sum_{\substack{p \leqslant x \\ M M_{0} \| f(p)}} \log p=x\left(P_{l}(\log y)+O(E(y))+O\left(x(\log x)^{-A}\right)\right.
$$

which is Theorem 1.1 for this case. 
Case 2: $1=r_{1} \leqslant r_{2} \leqslant \ldots \leqslant r_{l}$

In this case (2.5) is not relevant so we proceed in a different way using the remark after the proof of Lemma 2.3. By (4.16) and (4.17)

$$
\begin{aligned}
\sum_{\substack{p \leqslant x \\
M M_{0} \| f(p)}} \log p & =\sum_{\substack{k\left|M \\
k_{0}\right| M_{0}}} \mu(k) \mu\left(k_{0}\right) \sum_{j=1}^{\lambda(M k) \lambda\left(M_{0} k_{0}\right)} \Psi\left(x ; b_{j}, M k M_{0} k_{0}\right)+O(\sqrt{x}) \\
& =x \sum_{\substack{k\left|M \\
k_{0}\right| M_{0}}} \mu(k) \mu\left(k_{0}\right) \frac{\lambda(M k)}{\varphi(M k)} \frac{\lambda\left(M_{0} k_{0}\right)}{\varphi\left(M_{0} k_{o}\right)}+\mathcal{E}\left(x ; M, M_{0}\right) \\
& =x \frac{\rho(m)}{m} C\left(M_{0}\right)+\mathcal{E}\left(x ; M, M_{0}\right)
\end{aligned}
$$

on using (2.6), (4.4), (5.3) and noting that $\sum_{k \mid M} \frac{\mu(k)}{\varphi(M k)}=\frac{1}{M}$.

With $\chi$ a character $\left(\bmod M k M_{0} k_{0}\right)$, we have using (4.3) and (4.4) that

$$
\begin{aligned}
& \mathcal{E}\left(x ; M, M_{0}\right) \\
& \ll \sum_{\substack{k\left|M \\
k_{0}\right| M_{0}}}\left|\mu(k) \mu\left(k_{0}\right)\right| \lambda(M k) \lambda\left(M_{0} k_{0}\right) \\
& \quad \times\left\{\frac{1}{\varphi\left(M k M_{0} k_{0}\right)}\left(\sum_{\chi}^{*}|\Psi(x ; \chi)|+x E(x)\right)+\left(\log \left(x M k M_{0} k_{0}\right)\right)^{2}\right\} \\
& \quad+\sqrt{x} .
\end{aligned}
$$

We now want to sum (5.10) and (5.11) over $M M_{0} \leqslant y$, and we have by (5.4) that the main term is $x\left(P_{l}(\log y)+O(E(y))\right.$ as required. We split the sum of the error term into three parts as in case 1 . Note that $k \leqslant \prod_{q \mid m} q \leqslant m \leqslant M$ for $r_{1}=1$ and that the sum over $k$ has $2^{\omega(m)}$ terms. When $M M_{0} \leqslant y$ we can certainly say that $M k M_{0} k_{0} \leqslant y^{2}$ for each squarefree $k\left|M, k_{0}\right| M_{0}$. Moreover given $K \leqslant y^{2}, \quad K=M k M_{0} k_{0}$ in at most $2^{\omega\left(m m_{0}\right)}$ ways.

(i) By (2.6), (2.7) and (3.14) $\lambda(M k) \lambda\left(M_{0} k_{0}\right) 2^{\omega\left(m m_{0}\right)} \ll\left(M M_{0}\right)^{1-\delta}$ where $\delta=\frac{1}{l r_{l}}-\varepsilon$. Hence with $\chi$ a character $(\bmod K)$ and $Y=y, \quad Y^{3-\delta} \leqslant$ $x^{1 / 2}(\log x)^{-A-4}$ we deduce from (4.14) that

$$
\begin{gathered}
\sum_{M M_{0} \leqslant y} \sum_{\substack{k\left|M \\
k_{0}\right| M_{0}}}\left|\mu(k) \mu\left(k_{0}\right)\right| \lambda(M k) \lambda\left(M_{0} k_{0}\right) \frac{1}{\varphi\left(M k M_{0} k_{0}\right)} \sum_{\chi}^{*}|\Psi(x ; \chi)| \\
\ll \sum_{K \leqslant Y^{2}} \frac{\min \left(K^{1-\delta}, Y^{1-\delta}\right)}{\varphi(K)} \sum_{\chi}^{*}|\Psi(x ; \chi)| \ll x(\log x)^{-A} .
\end{gathered}
$$

This holds even when $l r_{l}=1$. 
(ii)

$$
\begin{aligned}
\sum_{M M_{0} \leqslant y} \sum_{\substack{k\left|M \\
k_{0}\right| M_{0}}}\left|\mu(k) \mu\left(k_{0}\right)\right| & \frac{\lambda(M k)}{\varphi(M k)} \frac{\lambda\left(M_{0} k_{0}\right)}{\varphi\left(M_{0} k_{o}\right)} \\
& \ll \sum_{M M_{0} \leqslant y} \frac{\rho(m)}{m} \prod_{q \mid m} \frac{1+\frac{1}{q}}{1-\frac{1}{q}} M_{0}^{-\frac{1}{l r_{l}}+\varepsilon} \\
& \ll(\log y)^{l}
\end{aligned}
$$

on using (2.6), (2.7) and (3.12).

(iii)

$$
\begin{aligned}
& \sum_{M M_{0} \leqslant y} \sum_{\substack{k\left|M \\
k_{0}\right| M_{0}}}\left|\mu(k) \mu\left(k_{0}\right)\right| \lambda(M k) \lambda\left(M_{0} k_{0}\right)\left(\log \left(x M k M_{0} k_{0}\right)\right)^{2} \\
& \ll(\log (x y))^{2} \sum_{M M_{0} \leqslant y} M \frac{\rho(m)}{m} 2^{\omega(m)} M_{0}^{1-\frac{1}{l r_{l}}+\varepsilon} \\
& \ll y(\log y)^{2 l-1}(\log (x y))^{2}
\end{aligned}
$$

by $(3.13)$.

From equations (5.11) to (5.14) we deduce

$$
\begin{aligned}
& \sum_{M M_{0} \leqslant y}\left|\mathcal{E}\left(x ; M, M_{0}\right)\right| \\
& \ll x(\log x)^{-A}+x E(x)(\log y)^{l}+y(\log y)^{2 l-1}(\log (x y))^{2}+y \sqrt{x} \\
& \ll x(\log x)^{-A}
\end{aligned}
$$

provided (1.4) holds. Then it follows from (5.10), (5.15) and (5.4) that (5.9) holds in this case. This completes the proof of Theorem 1.1.

Proof of Corollary 1.2. This follows from the formula

$$
\pi(x ; b, K)=\frac{1}{\varphi(K)} l i(x)+O\left(\max _{n \leqslant x,(b, K)=1}\left|\Psi(n ; b, K)-\frac{n}{\varphi(K)}\right|+\sqrt{x}\right) .
$$

To establish this, substitute $\Lambda(n)=\Psi(n ; b, K)-\Psi(n-1 ; b, K)$ in $\pi(x ; b, K)=$ $\sum_{\substack{2 \leqslant n \leqslant x \\ n \equiv b(\bmod K)}} \frac{\Lambda(n)}{\log n}+O(\sqrt{x})$ and then use partial summation, subtract $\frac{n}{\varphi(K)}$ from

$\Psi(n ; b, K)$ and compensate; then use partial summation again to obtain the main term. We can now use (5.16) in an argument analogous to the two cases of the proof of Theorem 1.1 and we obtain the same polynomial $P_{l}(\log y)$ and an error term involving the same estimate of $\sum_{M M_{0} \leqslant y}\left|\mathcal{E}\left(x ; M, M_{0}\right)\right|$ in the result. 


\section{Sketch proof of Theorem $\mathbf{1 . 3}$}

As in Lemma $2(\mathrm{i})$ write $\alpha=\left(t_{i}-1\right) r_{i}+\beta_{i}$ with $1 \leqslant \beta_{i} \leqslant r_{i}$ and assume $q \mid M_{i} \Rightarrow$ $q>p_{0}$. Then

$$
M_{i}:=\prod_{q^{\alpha} \| M_{i}} q^{\alpha}\left|f_{i}^{r_{i}}(n) \Longleftrightarrow m_{i}:=\prod_{\substack{q^{\alpha} \| M_{i} \\ t_{i}=\left\lceil\frac{\alpha}{r_{i}}\right\rceil}} q^{t_{i}}\right| f_{i}(n) .
$$

Note that $m_{i} \geqslant M_{i}^{1 / r_{i}} \geqslant M_{i}^{1 / r_{l}}$. With $M=\prod_{i=1}^{l} M_{i}, \quad m=\prod_{i=1}^{l} m_{i}$ we have

$$
M|f(n) \Longleftrightarrow m| f_{0}(n), \quad \frac{M}{m} \leqslant M^{1-\frac{1}{r_{l}}} .
$$

By (2.2), (2.4), (2.6) and (3.14)

$$
\lambda(M)=\frac{M}{m} \rho(m)<M^{1-\frac{1}{r_{l}}+\varepsilon} \leqslant M^{1-\frac{1}{l_{l}}+\varepsilon} .
$$

By $(2.3),(2.4)$ and $(2.7) \lambda\left(M_{0}\right) \ll M_{0}^{1-\frac{1}{l r_{l}}+\varepsilon}$.

Denote the residue classes $\left(\bmod M M_{0}\right)$ for which $M M_{0} \mid f(n)$ by $a_{j}, j=$ $1, \ldots, \lambda(M) \lambda\left(M_{0}\right)$. Then

$$
\begin{aligned}
\sum_{M M_{0} \leqslant y} \sum_{\substack{n=p^{\alpha} \leqslant x \\
M M_{0} \mid f(p)}} \Lambda(n) & =\sum_{M M_{0} \leqslant y}\left\{\sum_{j=1}^{\lambda(M) \lambda\left(M_{0}\right)} \Psi\left(x ; a_{j}, M M_{0}\right)+O(\sqrt{x})\right\} \\
& =x \sum_{M M_{0} \leqslant y} \frac{\lambda(M) \lambda\left(M_{0}\right)}{\varphi(M) \varphi\left(M_{0}\right)}+O\left(\sum_{M M_{0} \leqslant y}\left|\mathcal{E}\left(x ; M, M_{0}\right)\right|\right)
\end{aligned}
$$

where for $\chi$ a character $\left(\bmod M M_{0}\right)$ we have by (4.2), (4.3) and (4.4)

$$
\begin{aligned}
\mathcal{E}(x ; & \left.M, M_{0}\right) \\
& \ll \frac{\lambda(M) \lambda\left(M_{0}\right)}{\varphi(M) \varphi\left(M_{0}\right)}\left\{\sum_{\chi}^{*}|\Psi(x ; \chi)|+x E(x)\right\}+\left(\log \left(x M M_{0}\right)\right)^{2} \lambda(M) \lambda\left(M_{0}\right) \\
& +\sqrt{x} .
\end{aligned}
$$

The main term in (6.2) is obtained from Lemma 3.2 on using the generating functions

$$
\begin{aligned}
F(s) & =\sum_{M} M \frac{\lambda(M)}{\varphi(M)} M^{-s}=\prod_{i=1}^{l} \prod_{q \geqslant p_{0}}\left\{1+\frac{q \rho(q)}{q-1} \sum_{\beta_{i}=1}^{r_{i}} \sum_{t_{i}=1}^{\infty} q^{-t_{i}-\left(\left(t_{i}-1\right) r_{i}+\beta_{i}\right)(s-1)}\right\} \\
& =\prod_{i=1}^{l} \prod_{\beta_{i}=1}^{r_{i}} \zeta_{i}\left(\beta_{i}(s-1)+1\right) H(s)
\end{aligned}
$$


where $H(s)$ is analytic in $\sigma>1-\frac{1}{2 r_{l}}$, and $G(s)=\sum_{M_{0}} M_{0} \frac{\lambda\left(M_{0}\right)}{\varphi\left(M_{0}\right)} M_{0}^{-s}$ which is analytic in $\sigma>1-\frac{1}{l r_{l}}+\varepsilon$. Since $F(s)$ has a pole at $s=1$ of order $\sum_{i=1}^{l} r_{i}=R$, we deduce from (3.6) and (3.7) that

$$
\sum_{M M_{0} \leqslant y} \frac{\lambda(M) \lambda\left(M_{0}\right)}{\varphi(M) \varphi\left(M_{0}\right)}=P_{R}(\log y)+O(E(y))
$$

where the leading coefficient of $P_{R}$ is

$$
\frac{1}{R !} \prod_{i=1}^{l} \frac{\lambda_{i}^{r_{i}}}{r_{i} !} H(1) G(1)
$$

By (2.7), (6.1), (6.3), Lemma 4.5 and an argument similar to that in section 5 it follows that

$$
\sum_{M M_{0} \leqslant y}\left|\mathcal{E}\left(x ; M, M_{0}\right)\right| \ll x(\log x)^{-A}
$$

provided $\Delta$ and $y$ satisfy the conditions given in Theorem 1.3.

Theorem 1.3 now follows from (6.2), (6.5) and (6.7) with (6.6) giving the leading coefficient of $P_{R}$.

\section{Outline proof of Theorem 1.4}

(i)(a) Suppose $r_{1}=\ldots=r_{j}<r_{j+1} \leqslant \ldots \leqslant r_{l}$ for some $j(0 \leqslant j \leqslant l)$, and let $g=\prod_{i=1}^{j} f_{i}, h=\prod_{i=j+1}^{l} f_{i}{ }^{r_{i}}$. If $M \mid f(n)$ then $M=M_{g} M_{h}$ with $M_{g}\left|g(n), M_{h}\right| h(n)$, $\left(M_{g}, M_{h}\right)=1$, so $M_{g}=\prod_{i=1}^{j} M_{i}=m_{g}, M_{h}=\prod_{i=j+1}^{l} M_{i}, m_{h}=\prod_{i=j+1}^{l} m_{i}, m=m_{g} m_{h}$ on using our previous notation. Define $\lambda_{h}^{*}\left(M_{h}\right), \lambda_{h}\left(M_{h}\right), \lambda_{g}\left(M_{g}\right), \lambda\left(M_{0}\right)$ in an analogous way to $(2.4)$, and then by $(2.5),(2.6),(2.7)$

$$
\begin{array}{ll}
\lambda_{h}^{*}\left(M_{h}\right)=\varphi\left(M_{h}\right) \frac{\rho_{h}\left(m_{h}\right)}{m_{h}}, & \lambda_{h}\left(M_{h}\right)=\frac{M_{h}}{m_{h}} \rho_{h}\left(m_{h}\right), \\
\lambda_{g}\left(M_{g}\right)=\lambda_{g}\left(m_{g}\right)=\rho_{g}\left(m_{g}\right), & \lambda\left(M_{0}\right) \ll M_{0}^{1-\frac{1}{l r_{l}}+\varepsilon}
\end{array}
$$

and $\rho_{f}(m)=\rho_{g}\left(m_{g}\right) \rho_{h}\left(m_{h}\right)$.

Suppose $k_{g}\left|m_{g}, \quad k_{0}\right| M_{0}$ are squarefree. In order to consider when $M M_{0} \| f(n)$ we use ideas from case 2 in the proof of Theorem 1.1. We see that

$$
\begin{array}{r}
\#\left\{n\left(\bmod M_{h} m_{g} k_{g} M_{0} k_{0}\right): M_{h} \| h(n), m_{g} k_{g}\left|g(n), M_{0} k_{0}\right| f(n)\right\} \\
=\lambda_{h}^{*}\left(M_{h}\right) \lambda_{g}\left(m_{g}\right) \lambda\left(M_{0} k_{0}\right)
\end{array}
$$


since $\lambda_{g}\left(m_{g} k_{g}\right)=\lambda_{g}\left(m_{g}\right)=\rho_{g}\left(m_{g}\right)$. Then by the inclusion-exclusion principle we have on using (7.1) and (7.2) that

$$
\begin{aligned}
& \#\left\{n \leqslant x: M_{h}\left\|h(n), m_{g}\right\| g(n), M_{0} \| f(n)\right\} \\
& =\lambda_{h}^{*}\left(M_{h}\right) \sum_{k_{g} \mid m_{g}} \mu\left(k_{g}\right) \lambda_{g}\left(m_{g} k_{g}\right) \sum_{k_{0} \mid M_{0}} \mu\left(k_{0}\right) \lambda\left(M_{0} k_{0}\right)\left(\frac{x}{M_{h} m_{g} k_{g} M_{0} k_{0}}+O(1)\right) \\
& =x \frac{\rho_{f}(m)}{m} \prod_{q \mid m}\left(1-\frac{1}{q}\right) \frac{\varphi\left(M_{0)}\right.}{M_{0}} C\left(M_{0}\right)+O\left(\rho_{f}(m) \frac{\varphi\left(M_{h}\right)}{m_{h}} 2^{\omega\left(m_{g}\right)} M_{0}^{1-\frac{1}{l r_{l}}+\varepsilon}\right)
\end{aligned}
$$

where $C\left(M_{0}\right)$ is defined in (5.3). On summing (7.3) over $M M_{0} \leqslant y$ and using arguments similar to those in (3.8) and (3.13) we establish Theorem 1.4(i)(a) that

$$
\sum_{M M_{0} \leqslant y} \#\left\{n \leqslant x: M M_{0} \| f(n)\right\}=x\left(P_{l}(\log y)+O(E(y))\right)+O\left(y(\log y)^{l+j-1}\right) .
$$

The leading coefficient of $P_{l}$ is similar to (3.11) except that the values of $H_{1}(1)$, $G_{1}(1)$ are marginally different. This establishes (i)(a).

To derive (i)(b) let $Y=y(\log y)^{-j}$. Then by (i)(a)

$$
\begin{aligned}
\sum_{M M_{0} \leqslant Y} \#\left\{n \leqslant x: M M_{0} \| f(n)\right\} & =x\left(P_{l}(\log Y)+O(E(Y))+O\left(Y(\log Y)^{l+j-1}\right)\right. \\
& =x\left(B_{l}(\log y)^{l}+O\left((\log y)^{l-1} \log \log y\right)\right) .
\end{aligned}
$$

With $M=\prod_{i=1}^{l} M_{i}, M_{i}^{1 / r_{i}} \in \mathbb{N}$ as in section 6 and using an argument similar to that used to prove Theorem 1.4(ii) we have

$$
\begin{aligned}
\sum_{Y<M M_{0} \leqslant y} \#\{n \leqslant x & \left.: M M_{0} \| f(n)\right\} \\
& \leqslant \sum_{Y<M M_{0} \leqslant y} \#\left\{n \leqslant x: M M_{0} \mid f(n)\right\} \\
& =x\left(Q_{l}(\log y)-Q_{l}(\log Y)+O(E(y))+O\left(y(\log y)^{l-1}\right)\right. \\
& \ll x(\log y)^{l-1} \log \log y
\end{aligned}
$$

where $Q_{l}($.$) is a polynomial of degree l$. The result of (i)(b) follows.

(ii) Defining $M, m$ as in section 6 , by (6.1) and an argument similar to (6.5) and (6.6) we deduce Theorem 1.4(ii) that

$$
\begin{aligned}
\sum_{M M_{0} \leqslant y} \#\{n \leqslant x: & \left.M M_{0} \mid f(n)\right\} \\
& =x \sum_{M M_{0} \leqslant y} \frac{\rho(m)}{m} \frac{\lambda\left(M_{0}\right)}{M_{0}}+O\left(\sum_{M M_{0} \leqslant y} \frac{M}{m} \rho(m) \lambda\left(M_{0}\right)\right) \\
& =x\left(P_{R}(\log y)+O(E(y))\right)+O\left(y(\log y)^{R-1}\right)
\end{aligned}
$$

where the leading coefficient of $P_{R}$ is similar to that in (6.6) but with slightly different values for $H(1), G(1)$. 
To deduce the Corollary 1.5 from (i)(b) for $f$ an irreducible quadratic, let $X=\left(\sup _{n \leqslant x}|f(n)|\right)^{1 / 2} \times x$. To each exact divisor $M>X$ of $f(n), \frac{f(n)}{M}$ is an exact divisor $<X$. Hence

$$
\sum_{M} \sum_{\substack{n \leqslant x \\ M \| f(n)}} 1=2 \sum_{M \leqslant X} \sum_{\substack{n \leqslant x \\ M \| f(n)}} 1-\sum_{M \leqslant X} \sum_{\substack{n \leqslant x \\ M \| f(n) \\ \frac{|f(n)|}{M} \leqslant X}} 1 .
$$

The first double sum on the right of (7.4) equals $2 B_{1} x \log x+O(x \log \log x)$ by (i)(b). Since $|f(n)| \geqslant c n^{2}$ for some $c>0$, the second double sum on the right of (7.4) is

$$
\leqslant \sum_{M \leqslant X} \sum_{\substack{n \leqslant \sqrt{\frac{X M}{c}} \\ M \mid f(n)}} 1 \leqslant \sum_{M \leqslant X} \rho(M)\left(\sqrt{\frac{X}{c M}}+O(1)\right) \ll x .
$$

Corollary 1.5 now follows from (7.4).

\section{References}

[1] H. Davenport, Multiplicative Number Theory, $3^{\text {rd }}$ ed., revised by H.L. Montgomery, Springer, New York, 2000.

[2] P. Erdős, On the sum $\sum_{k=1}^{x} d(f(k))$, J. London Math. Soc. 27 (1952), 7-15.

[3] H. Halberstam, Footnote to the Titchmarsh-Linnik Divisor Problem, Proc. Amer. Math. Soc. 18 (1967), 187-188.

[4] G. Hanrot, G. Tenenbaum. J. Wu, Moyennes de certaines fonctions multiplicatives sur les entiers friables 2, Proc. London Math. Soc. (3) 96 (2008), $107-135$.

[5] C. Hooley, On the number of divisors of quadratic polynomials, Acta Math. 110 (1963), 97-114.

[6] J. McKee, On the average number of divisors of quadratic polynomials, Math. Proc. Camb. Phil. Soc. 117 (1995), 389-392.

[7] J. McKee, A note on the number of divisors of quadratic polynomials, Sieve methods, exponential sums, and their application in number theory, LMS Lecture Note Series 237, G. Greaves, G. Harman, M. N. Huxley (eds.), CUP, Cambridge, 1997, 275-281.

[8] J. McKee, The average number of divisors of an irreducible quadratic polynomial, Math. Proc. Camb. Phil. Soc. 126 (1999), 17-22.

[9] T. Nagell, Introduction to Number Theory, $2^{\text {nd }}$ ed., Chelsea, New York, 1964.

[10] I. Niven, H. S. Zuckerman, H. L. Montgomery, An Introduction to the Theory of Numbers, $5^{\text {th }}$ ed., Wiley, New York, 1991.

[11] G. Rodriguez, Sul problema dei divisori di Titchmarsh, Boll. Un. Mat. Ital. (3) 20 (1965), 358-366. 
[12] E.J. Scourfield, The divisors of a quadratic polynomial, Proc. Glasgow Math. Assoc. 5 (1961), 8-20.

[13] E.J. Scourfield, Smooth Divisors of Polynomials, Number Theory and Polynomials, LMS Lecture Note Series 352, J. McKee, C. Smyth (eds.), CUP, Cambridge, 2008, 286-311.

[14] G. Tenenbaum, Sur une question d'Erdôs et Schinzel, A Tribute to Paul Erdôs, A. Baker, B. Bollobás, A. Hajnal (eds.), CUP, Cambridge, 1990, 405-443.

[15] R.C. Vaughan, An elementary method in prime number theory, Acta Arith. 37 (1980), 111-115.

Address: Eira J. Scourfield: Mathematics Department, RHUL, Egham, Surrey TW20 0EX, UK. E-mail: e.scourfield@rhul.ac.uk

Received: 11 August 2015; revised: 27 January 2016 